\title{
Analytics of biometric data from wearable devices to support teaching and learning activities
}

\author{
Francisco de Arriba Pérez , Juan Manuel Santos Gago \& Manuel Caeiro Rodríguez \\ ${ }^{1}$ Universidad de Vigo, SPAIN
}

\begin{abstract}
This paper introduces the preliminary results of a piece of research whose main purpose is to take advantage of data collected from wearable devices to support learning processes. This goal is approached through the application of learning analytic techniques. The innovation point is the use of data collected from wearables, that will be used in conjunction with data collected from other sources (e.g. Learning Management Systems, Student Information Systems). The paper reviews the results achieved during the last year about the relationships among biometric data collected from wearables and relevant features described in the educational literature. In this way sleep and stress have been identified as interesting areas that could be informed from data collected in wearables and processed by applying machine learning techniques. Our preliminary results show some initial promising results that need further validation, also these results show an interesting opportunity to support awareness and intervention functionalities.
\end{abstract}

\author{
Keywords \\ learning analytics, \\ wearable sensors, \\ wearable computing, \\ smartphones sensors, \\ smartphones computing, \\ sleep detection, \\ stress detection \\ Received: 11 Jan 2016 \\ Revised: 26 Jan 2016 \\ Accepted: 21 Feb 2016 \\ DOI: $10.20897 /$ lectito. 201608
}

\section{INTRODUCTION}

Currently, expressions such as the following ones are not strange: "The speed of the digital transformation is creating new ecosystems, actors and even industries that offer everyone a huge potential" (ITU Telecom World 2015, 2015). These technologies can be found to a large extend in many of the commercial and industrial activities, event in the daily activities of the common people (whether funny, educational or workrelated), mainly in the most developed countries. Nowadays, many ordinary activities are supported by ICTs, particularly, by telematics application and services. This scene has been strengthened in a big way since during the last decade since the advanced phone devices have become mainstream, such as smartphones, tablets, or more recently, the burst into the market of the smartbands and smartwatches as wearable devices involving more users from day to day. In the recent years these devices, in conjunction to the evolution and the decrease in price of the itinerant communication technologies (e.g. LTE and 4G networks), have enabled the development of new ways to use telematics services beyond the desktop computers.

The growing proliferation of telematics services in the daily activities has given rise to the availability of digital data associated to different activity types generated in the devices. Such data can be easily processed by machines, and therefore, they can be exploited properly to provide several types of high added value applications. In this way, during the last years, an explosion has been produced related to applications that make use of techniques from the Data Analytics field (Russom and others, 2011) (or some of its variations, such as Data Mining (Romero and Ventura, 2010, Fayyad et al., 1996), Machine Learning (Bratko, Michalski and Kubat, 1999), Business Intelligence (Chen, Chiang and Storey, 2012), Big Data (Manyika et al., 2011, Mayer-Schönberger and Cukier, 2013)). These techniques try to come up with some information, or even 
knowledge, from the analysis of the data collected in some particular context. Such information may be used to provide personalized or customized services to users and companies in many varied contexts.

The education and learning area is not unaware of this situation, definitely. TICS have been introduced in this area since nearly two decades ago. TICs have been considered as a tool that may

contribute to universal access to education, equity in education, the delivery of quality learning and teaching, teachers' professional development and more efficient education management, governance and administration. (UNESCO, 2015)

Therefore, multitude of organizations, as the UNESCO itself, have promoted and supported the introduction of technologies into educational environments. In this way, Learning Management Systems (LMS) have evolved and become popular to the point there are really extended at all levels of the modern societies' educational systems. This fact has contributed to the availability of digital data related to learning activities (learner records and traces) that can be analyzed for purposes of understanding and optimizing learning and the environments in which it occurs (LAK2011, 2010). This specialization of data analytics applied to the educational area has given rise to a new discipline entitled Learning Analytics (LA).

As in other contexts, in the educational area, the appearance of advance mobile devices has given rise to new service paradigms. The Mobile Learning (m-learning) provides with a series of new advantages and benefits for technology-supported learning, such as:

- Greater ubiquity. The inherent mobility in these devices enables to break the physical and temporal barriers that limit the access to education to a greater extend.

- Greater interaction among users. The use of mobile devices promotes the communication (both formal and mainly informal) among learners and teachers.

- New learning methods. The use of techniques such as geolocation or augmented reality open a new framework for learning. Contents do not need to be physical entities order to be handled by learners, any activity may be dynamic and contextualized to the place where the learner is located, and it can be supported in videos/animations to improve learning, in a fast and simple way.

Therefore, mobile technologies are called to become popular in learning environments. In fact, despite we should consider this is at an embryonic state yet, this popularization has already been started. As in other areas, this fact result in a greater amount of data, even the emergence of new data types, that can be analyzed to extract information to improve the learning activities and the performance of the involved stakeholders.

The work introduced in this paper belongs to the Learning Analytics field. In this discipline, significant advances have been produced during the last years, as reflected in several state of the art reviews (Baker and Inventado, 2014, Romero and Ventura, 2013). The authors of this work have taken part and continue taking part actively in projects of this discipline during the last 3 years. Currently, one of the research lines they are promoting is oriented towards the information and knowledge extraction from data generated in sensors of mobile devices and, mainly, of wearables such as smartwatches used by learners (not necessarily just during sport activities, but everywhere and in every moment). This research line has received little attention in the Learning Analytics field up to date and, presumably, it has a great potential towards the achievement of enhancement goals, involving not just academic activities but also biometrical parameters that feature mood, physical and mental conditions of the learner. The description of the work introduced here extends and updates the communication (de Arriba Perez, Caeiro Rodriguez and Santos Gago, 2015) published in the 10th Iberian Conference on Information Systems and Technologies.

The paper is organized as follows. This first section introduces the context and motivation of the work. Next section, characterize the goals. Sections 3, 4 and 5 introduce the results achieved to date corresponding to the review of wearable devices, the identification of indexes to feature learners based on data collected from mobile devices used by students in a daily basis and the system architecture that supports to perform the analytics with the collected data. To finish, section 6 introduces the conclusions obtained from the work performed and lists the actions that will be carried out to continue the initiated work.

\section{OBJECTIVES}

The general goal of this work is the development of a framework to facilitate the development of systems oriented to extract information and knowledge in a holistic way from data generated in specific educational contexts. Nowadays, in the Learning Analytics area there exist many proposals focused on the extraction of this type of information based on the data generated by the software applications available in Learning Management Systems (LMSs) used by learners to perform their educational tasks, in the Student Information 
Systems (SIS) used by school administrators, and other academic systems. Nevertheless, up to date, proposals involving other data sources have not been common. Particularly, there have not been many proposals involving the use of data generated by wearable devices, such as smartbands and smartwatches.

Indeed, smartbands and smartwatches are in an early stage of adoption in the electronic consumer market yet. Nevertheless, they have already stopped being strangers for the common people and now there exist applications taking advantage of these devices for, based on the analytics of their captured data, provide personalized services to users. The most popular applications are electronic trainers, probably. These applications allow, for example, to generate training (or maintenance) plans to users based on the physical condition and evolution. This type of applications has received a good acceptance in the market, mainly among young people, and in this way they have promoted the quick popularization of wearables. More and more every day people is adopting wearables in daily activities.

Based on this scenario we have initiated a piece of research oriented towards the exploitation of wearable generated data in young students to get information that can be used to complement data obtained from other systems (e.g. SIS, LMS) to feature learners, educational resources and academic processes. Some of the data provided by wearables interesting for our piece of research are: heart rate, body temperature, ambient luminosity, localization or instantaneous movements. This data can be used to obtain information such as the level of attention during the performance of academic activities, stress level or attention during homework, etc. This information can be especially useful from two perspectives:

- To detect students at risk and difficulties, or as in the case of electronic trainers, to provide a personal task plan, to recommend activities according to the tiredness or stress, to suggest some rest or leisure activity if symptoms of fatigue are observed after a prolonged hard work, etc.

- To assess the effectiveness and efficiency of particular educational contents or educational activities. For example, during a lesson the moments when the students pay more attention could be identified. Or on the contrary, the moments when the students are more disoriented. This information can be useful to improve the educational resources and approaches involved.

To achieve this generic goal, we have plan several partial goals, smaller in magnitude, to approach the work in a more systematic way. Specifically, the partial set goals are:

1. To perform a study of the wearable devices available in the market currently. This study should include: i) an analysis of the evolution and introduction degree of the devices in the market, ii) a study of the features of the devices and of the sensors included and iii) a study of the access options and the mechanisms available to collect data generated in the sensors.

2. To identify indexes to be used as learner features. Clearly, the raw values of variables such as heart rate or instantaneous movement for a learner are not very useful information. This data need to be processed and analyzed to get patterns or indexes at an upper level, such as the stress or somnolence level. These indexes can be used as learner features, namely, they allow to complement traditional learner models (including indicators such as academic performance and demographic data) and to analyze changes and evolution. Obviously, indexes that can be calculated from the collected data in wearables need to be identified and the methodology to calculate such indexes needs to be established. In many cases, Machine Learning techniques will play a main role.

3. To define a reference architectonic model to develop applications oriented to provide particular services to the users based on the data collected from wearables and other systems. This architecture should take into account the several inherent conditions involved in the data collection depending on the wearable providers.

4. To test the utility of the solutions in specific educational scenarios. Specifically, the development of recommendation and assistance applications to support two particular pedagogical approaches are planned: self-regulated learning and collaborative learning. Related to the first approach, the solutions developed can be really helpful as indexes of stress and motivation can be calculated. In the second case, we plan to develop an application to recommend to student groups the performance of shared tasks based on issues such as their coincidence at certain physical locations, the spare time available, or the similarities among their features. For example, some students work better on the mornings, while other one prefer to do tasks on the evenings.

Next sections briefly describe the main findings. 


\section{STUDY OF WEARABLES}

Before addressing the complex task of defining indicators based on biometric data and from the student academic context, it is essential to carry out a study of mobile devices, and more specifically, of the wearables that will be used to capture data. Particularly, next questions need to be answered:

1. What are wearables? What types of wearables are available on the market? The wearable term can be hearing more and more often, however, as usually happens with coined terms in the technology field, there is not a general accepted definition. We do not intend to find such definition, but we consider appropriate to precise the concept and, overall, to focus on the devices under this concept available on the market currently.

2. What is the degree of implementation of these wearables on the market? What is the forecast of their adoption? In our piece of research, we assume that in the short/medium term these devices will be adopted in a common way, mainly in the young people carrying out university studies. Therefore, it will be feasible to use them to collect biometric and contextual data about learners.

3. What data can be actually collected from these wearables at present or in the near future? How easy is to collect such data? A key issue in our piece of research is to know the sensors available in wearables because they provide the data needed to perform it. Another significant issue is related to features of the data and indicators, such as precision. However, this is not as important as in other fields (e.g. health care), because our purpose is basically to discover changes in the behavior of the students rather than on the absolute values of their biometric features. We do not attempt to find any sort of pathology at the person, but to detect situations in which the learner can feel nervous, stressed or issues such as lack of attention during a training session.

Related to the first question, what are the wearables? it is possible to find several definitions in the literature. One of the most popular meanings is given by Tehrani and Michael (Tehrani and Michael, 2014):

The terms 'wearable technology', 'wearable devices', and 'wearables' all refer to electronic technologies or computers that are incorporated into items of clothing and accessories which can comfortably be worn on the body.

However, from the perspective of our piece of research, it is particularly interesting the approach proposed by MIT researchers Crabtree y Rhodes (Crabtree and Rhodes, 1998) as early as in 1998:

The fuzzy definition of a wearable computer is that it's a computer that is always with you, is comfortable and easy to keep and use, and is as unobtrusive as clothing.

This definition emphasizes the ease of use of these devices, which can be carry by people transparently. In this way people can carry on and use them continuously, even when they are sleeping. This is very useful to collect certain data, such as the one related to the sleep quality or the chronotype. The same authors added a series of intrinsic features expected in a wearable device, among which we highlight:

A wearable should have sensors for the physical environment such as location (for example GPS), cameras, microphones, temperature, humidity, movement, etc.

This list of sensors can be extended with biometric signal sensors, such as Heart Rate (HR) or Galvanic Skin Response sensor (GSR), etc. In any case, these definitions could be applied to many different kinds of devices. Indeed, a plethora of devices, or at least prototypes, can be found in the market fitting more or less in the previous definitions, from small appliances that are built into the shoes sole or the insole to small devices that can be incorporated into users' glasses. Despite all these options, the more adopted wearables today, and the forecast for the near future, are wrist wearables, namely smartbands and smartwatches, this information is provided by (Richter, 2015) and it is show in Figure 1.

For the purposes of our piece of research we could use, to a greater or lesser extent, any of the wearable types available today. We do not rule out any of them, but taking into account their availability and adoption degree we focus in the wrist wearables mainly. In addition, these type of devices include sensors that are in contact with the skin permanently and therefore they can be used to collect in great detail different kinds of biometric data.

As mentioned before, another question that needs to be answered is related to the availability of sensors in the devices. The types of sensors usually included in wearables (mainly in wrist wearables) are key factors in order to develop a solution based on the results of our piece of research. We produced a list of available sensors in smartbands and smartwatches from the information available in recent publications (Swan, 2012) and (Rehman et al., 2015) and in several web portals. It is a complex task to find the real availability of each sensor type because the variety of devices and the continuous change. On the one hand the number of 
wearables is quite large and is changing every day. On the other hand, a sensor that is difficult to be found today, in some months can become very popular. Anyway, we do our best to provide a snapshot of the current situation related to the availability of sensors. We consulted specialized websites in wearables, the main sites of manufacturers. In total we have collected information about 145 different devices including its sensors, and with this information we build Figure 2.

As shown in the Figure 2, there are several highly available sensors that have the potential to be very useful to get biometric features. Obviously, some sensors that show a very low availability would be very

\section{The Predicted Wearables Boom Is All About The Wrist}

Worldwide wearable device shipment forecast (in million units)

$2014 \square 2015 \square 2019$

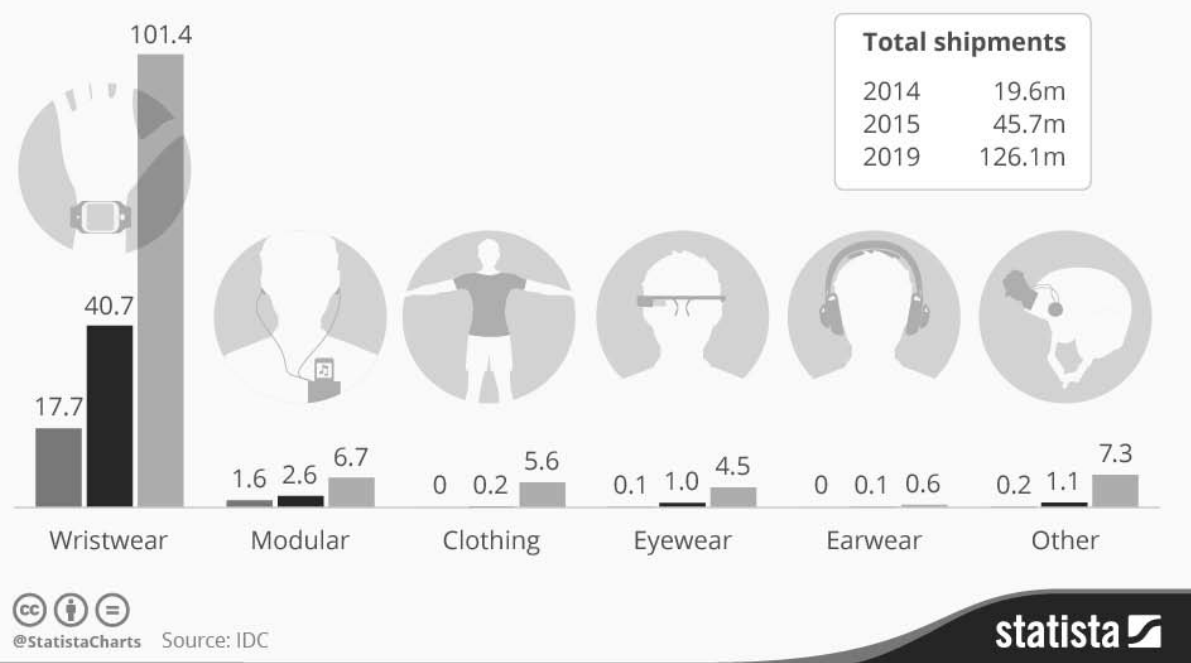

Figure 1. This chart shows a forecast of worldwide wearable device shipments (Richter, 2015)

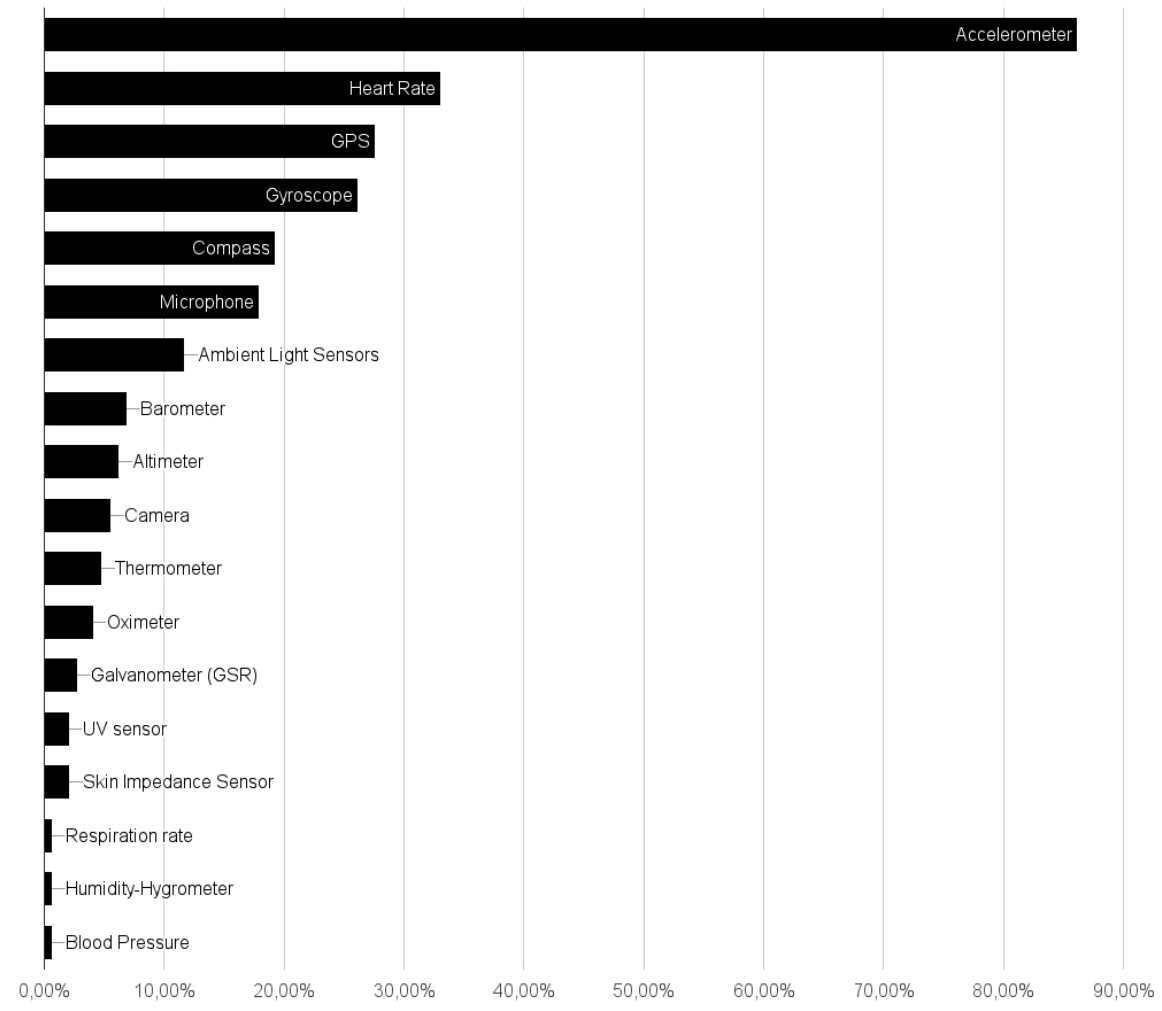

Figure 2. Percentage of sensors in wearables 
helpful, such as Blood Pressure, but just sensors as the accelerometer, HR and GPS can be used to provide interesting results towards our goals.

A final issue to consider in the analysis of wearable devices is related to the transfer of the collected data to other devices in order to process them. This issues could be considered as trivial. However, the devices available in the market today are largely limited in the options to get access to their collected data. Trying to provide a simple picture of the existing situation, two broad sets of approaches can be identified:

1. Access to raw data through a smartphone. This option included in some wearables allows to access to raw sensor data directly. Furthermore, this approach enables the researcher to perform real time studies. To transfer the data from the wearable to the smartphone a specific app needs to be developed and this requires programming knowledge about event communication, temporary storage to tackle with network outages, etc. Moreover, depending on the wearable (e.g. those devices based on Android Wear) an app in the wearable itself needs also be developed to act as a listener of the sensors.

2. Access to data provided by the wearable proprietary warehouse. Data is transferred through proprietary APIs based on REST services provided by the wearable provider. The main drawback of this approach is that real time access to data is not possible because it depends on the synchronization time between the wearable and the warehouse constantly. Such communication between the wearable and the warehouse is produced sporadically and not a regular time period as can be performed in the former approach. In some cases, the communication may take several days. Furthermore, in all the systems we analyzed the raw data is not provided, but processed information. For example, the nominal values of the accelerometer cannot be get from the warehouse, just derived data such as pedometer.

\section{INDICATORS FOR CHARACTERIZING THE BEHAVIOR OF STUDENTS}

The core of our piece of research involves the definition of indexes to feature the student from a biometric perspective, mainly. Learner registers (profiles) are used in the vast majority of learning environments including information about learning performance, demographic data and, in applicable, information about learning difficulties. The literature reports multitude of examples about many other information pieces proposed to enrich learner profiles. Some of them are used commonly, such as tracking logs and teacher evaluations, and other ones are more exotic, such as preferred learning styles. More recently, the development of the Educational Data Mining and Learning Analytics disciplines enlarged the range of learner features considered in the profiles considerably. These disciplines are specifically aimed to provide information from the automatic processing of raw data, such as educational tracking logs. Within this scope new learner features have been proposed related to the social behavior (where the Social Learning Analytics discipline has been developed (Shum and Ferguson, 2012)), motivation, cognitive model, engagement, etc.

Despite the significant recent developments, nowadays, to the best of our knowledge, there are not any relevant proposals involving the estimation of learners features based on biometric data. In particular, indicators inferred from analysis of biometric data collected in wearable devices carried on by learners have not been proposed yet. As it is introduced in section 2, this type of indicators can be really useful to improve the learning environments, both in relation to the learner performance as to optimization of the learning resources.

In this scenario, during the last 15 months we have been working towards the identification of indicators based on biometric data. In particular, we have focus our attention on two dimensions, one oriented to sleep features and another one oriented to stress features. Both dimensions have a clear influence on student performance as it is covered in the literature. In fact, a review of the available literature was performed as a first stage towards the identification of indicators on these dimensions.

\section{Sleep related indicators}

We reviewed the scientific literature as a first step towards the identification of sleep information items. As a result of this search we found a whole research field focused on sleep analysis, specially in medical and psychological areas. A question of interest in this field is related to the development of methods to measure and estimate indicators about the sleep quality. Two main approaches are observed: (i) based on subjective tests performed through the administration of questionnaires to the analysed subjects, and (ii) based on objective tests, monitoring biometrical features in lab contexts following very precise clinical protocols. As an example of the subjective approach we can reference the Epworth Test (Johns and others, 1991), which 
involves a questionnaire with 8 questions to measure the average daytime sleepiness. As an example of the objective approach we found the Multiple Sleep Latency Test (MSLT) (Carskadon et al., 1986), based on the analysis of certain factors of a person that is observed in a room while performing a certain number of micronaps. Logically, the objective test are usually more precise than the subjective ones, but they need much more resources to be carried out: time, appropriate equipped rooms, trained staff, specialized sensors (as an example, some tests require brain scans), etc.

In our case, the indicators should be calculated continuously and in a transparent way for the user. Basically, they need to be obtained in a similar way to the objective tests, but in a completely different environment, the learner natural contexts, without any interference in their common life. In addition, our equipment is not as sophisticated and precise as the devices used in the medical objectives tests, but we hope they will be valid enough to provide valuable data. Particularly, we consider that wearables can provide answers to some of the questions usually included in the subjective tests, such as "how many hours do you sleep at day?". Therefore, taking as reference the main indicators found in the literature and analyzing the factors involved in their calculation, we have come to a group of indicators that can be useful to achieve our goals. These are they indicators chosen:

- Quality of Sleep (QS). This indicator provides an estimation about how well the student has slept. It is based on the subjective test Pittsburgh Sleep Quality Index (PSQI) test (Buysse et al., 1989). From this test we have identified 5 variables that can be calculated from data collected in wearable sensors and can be used to estimate the QS according to the following formula:

$$
Q S=100 *\left(\frac{1}{10} * L S+\frac{1}{10} * T D+\frac{1}{10} * E f+\frac{1}{60} *(S T+A W)\right.
$$

o SL (Sleep Latency): It is a value between 0 and 3 that indicates the amount of time required to fall asleep.

o TD (Sleep Duration): It is a value between 0 and 3 that indicates the amount of time the person has slept.

$0 \quad$ EF (Efficiency): It is a value between 0 and 3 representing the relation between the effective time slept and the duration of the whole sleep period.

0 AW (Awakes): It is a value between 0 and 3 obtained from the number of times the student has awaken during the sleep period.

o ST (Skin Temperature): It is a value between 0 and 3 representing the average skin temperature.

We would like to highlight that the levels used to calculate each one of these factors and the weights included in the former formula have been taken from the PSQI procedure, directly.

- Sleepiness Level (SL). This indicator estimates the student drowsiness degree. To calculate it we use the values collected from the accelerometer and the HR sensors. When the accelerometer data indicates an average value over a certain threshold for 2 seconds, the drowsiness level is 0 (it is interpreted that the student is performing some movement). In other case, the drowsiness level is determined by the following expression:

$$
S(H R a)=\left\{\begin{aligned}
1, & H R a<0 \\
1-\left(\frac{H R a}{H R m i n}-1\right) / 0.2, & H R m i n<H R a \leq 1.2 * H R m i n \\
0, & H R a \geq 1.2 * H R m i n
\end{aligned}\right.
$$

o HRa is the average heart rate for a 20 seconds period

o HRmin is the heart rate in rest, namely, in conditions of maximum relax and comfort

- Chronotype (CR). This indicator features a subject based on the timing of the sleep cycles, which are closely linked to the circadian rhythm. The literature refers almost unanimously three main sleep cycles: (i) morning, referred to people whose cognitive functions are maximum in the morning and begin to decrease in the afternoon and therefore they prefer to go to sleep early and to wake up early; (ii) evening, referred to people who have a hard time to perform tasks involving high cognitive load in the morning, but their capabilities enhance during afternoon and evening; and (iii) the intermediate group, referred to people situated in the middle of the two former groups. The estimation of the chronotype can be performed based on the sleep behavior, despite actually this is not a trivial task. In our case we followed the classification proposed in the Horne and Östberg test (Horne and Ostberg, 1975), which involves 5 categories (cf. table 1) involving specific ranges for the start and end of the sleep periods. 
The classification of a subject in one of the categories is performed according to the time he goes to sleep and the time he wakes up. This data is taken just in non-working days, because it is assumed that such days he is free to lie down or to stand up when he prefers. Therefore, the first step towards the estimation of this feature is to identify periods of non-working days and to select days that fit in the middle of these periods. Then, as mentioned, the time at which the subject goes to sleep and wakes up are used to identify the chronotype category.

This algorithm is simple, but it provides acceptable results. Nevertheless, the data collected in working days is not used and they can become very significate. We are currently working in a more sophisticated version of the algorithm to take into account the data on such dates and also to estimate the degree of adherence of the subject to the category in which he is classified. This information seems very useful in order to design recommendations to students.

To validate the proposed indicators, we performed data collection from 4 participants ( 1 of the authors of this work, $2 \mathrm{PhD}$ students of the authors' research group and an external person) for a 15-day period. We developed a dashboard to analyze the results visually (cf. Figure 3). The participants were provided wearable devices from different vendors: Fitbit, Microsoft, Jawbone, etc. Furthermore, the participants also installed an app in their smartphones asking them about their sleep quality every morning as soon as they woke up

Table 1. Chorotype scale

\begin{tabular}{cll}
\hline Type & Start Sleep & End Sleep \\
\hline Definitely evening & $02: 00-03: 00$ & $10: 00-11: 30$ \\
Moderately evening & $00: 45-02: 00$ & $08: 30-10: 00$ \\
Neither & $22: 00-00: 45$ & $06: 30-08: 30$ \\
Moderately morning & $21: 30-22: 45$ & $05: 00-06: 30$ \\
Extreme morning & $21: 00-21: 30$ & $04: 00-05: 00$ \\
\hline
\end{tabular}
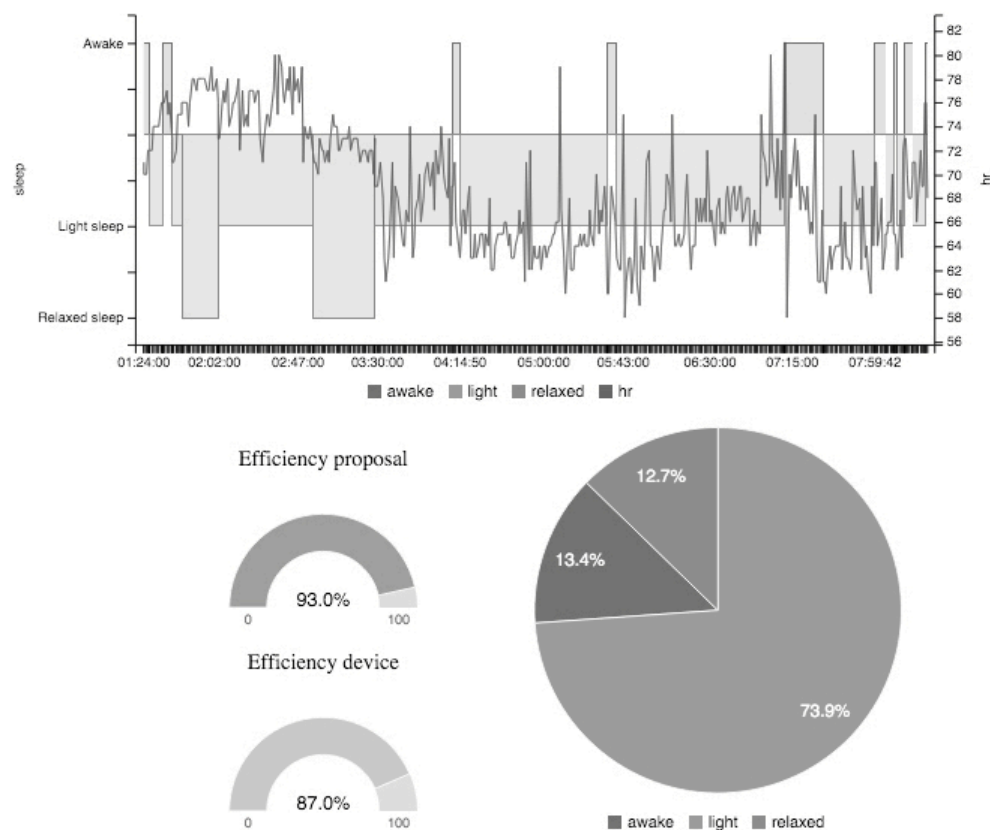

Data for principal Sleep Start hour: 01:24:04 End hour: 08:29:00 Total time: 07:04:56 Fall as sleep time: 00:03:28 Number of Awake: 7 Relaxed HR: 58 Temperature mean: 34.14

Summary of the last days 30 :
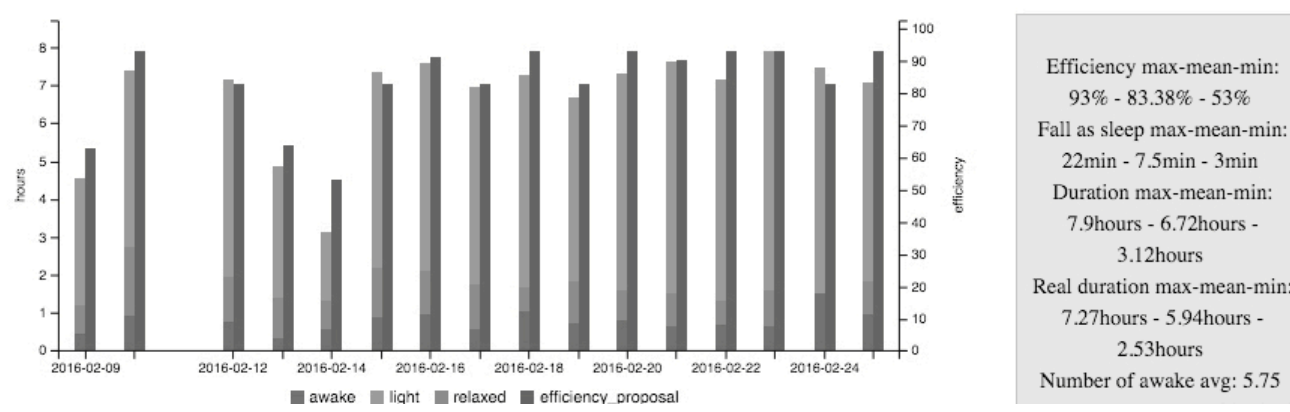

Figure 3. Dashboard of sleep 
(possible answers: bad, regular, well and very well). The first results of this indicator shows an improvement over the proposed Fitbit and Microsoft indicators. Our indicator modifies its value according to variations in sleep parameters. Despite this, we are in a phase of analysis so we can not provide a final result. Meanwhile, the validation of the SL was conducted asking to the participants to identify time periods when they felt highly relaxed (some participant identified this situation, for example, while they were watching a movie or after lunch in the weekend). The chronotype validation was performed taking into account the cases in which they went to sleep at night and wake up in the morning in a voluntary basis without any constraint of help (e.g. using an alarm clock).

\section{Stress related indicators}

In order to define indicators related to stress we also reviewed, once again, the existing literature to find out the different types of stress indexes and the ways in which they are calculated. In the vast literature available on this topic different kinds of stress have been documented according to their application context. In our case we are interested in stress produced in teaching and learning scenarios. In this way we find relevant the Burnout Syndrome (Maslach and Jackson, 1981) affecting to teachers both at university and compulsory schooling. This type of stress also occurs in children as indicated by recent studies (Pacheco, Durán and Rey, 2007), which also states that emotional intelligence is helpful to control it. An interesting result is that stress is not necessarily a bad thing. A certain level of stress improves attention and performance in the activities of a student. Typically, it is intended that the student is, when he/she is making an academic activity, in a specific state named as Eustress (cf. Figure 4 (Ávila, Garzón and Casallas, 2011)) where optimal performance is obtained.

The literature contains a number of tests based on questionnaires to estimate the stress level, such as the Burnout test (Maslach et al., 1997), Perceived Stress Scale (Cohen, Kamarck and Mermelstein, 1983) or ISRA (Antonio Cano-Vindel, Juan J Miguel-Tobal, 1999). Regretfully, these tests involve a high subjective component and the managed factors hardly can be estimated from biometric data. Fortunately, there are also many other proposals to estimate the stress level based on specific biometric data, such as GSR (Santos Sierra, 2012, Picard and Healey, 2000), HR (Santos Sierra, 2012) or skin temperature (Zhai and Barreto, 2006). Our proposal follows the approaches included in the latter work, but in a multidimensional way and replacing the devices (expensive and with reduced mobility) by wearables.

To estimate these indicators, we decided to apply Machine Learning traditional techniques directly to detect the weight to be applied to the factors involved. Our solution uses data collected from the accelerometer, HR and ST of the subjects during several weeks. Furthermore, the subjects are required to identify themselves how they feel during time periods daily, distinguishing among: low stress (for instance, watching TV, reading a book), medium stress (for instance, when they are performing some task that requires some attention such as driving), and high stress (for instance, presenting in classroom, during an exam). The tagging process was supported by a specific dashboard (cf. Figure 5). We collected around 4.000 samples (including accelerometer, HR and ST data for second) tagged in accordance to this classification to train several classification algorithms: J48, Bagging-J48, AdaBoost-J48, Bagging-IBk, RandomForest, IBk, NaiveBayes and OneR. In all cases the success rate was greater than $90 \%$.

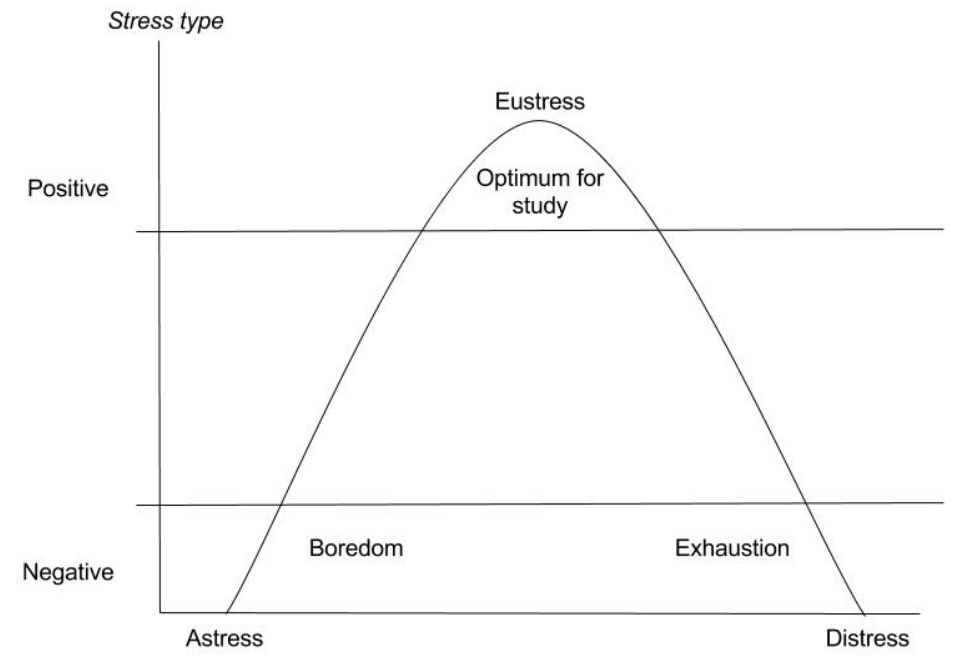

Figure 4. Stress graph (Ávila, Garzón and Casallas, 2011) 


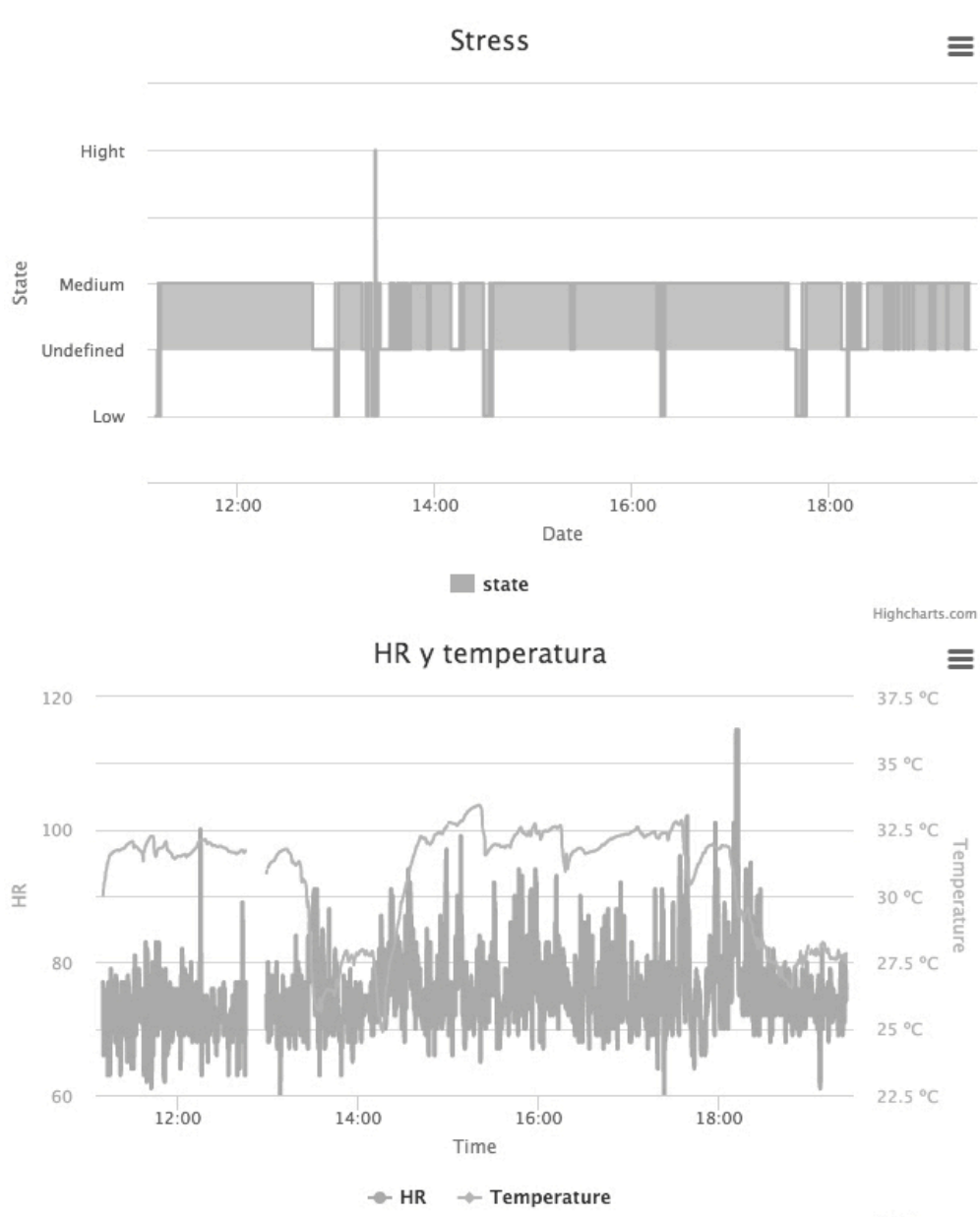

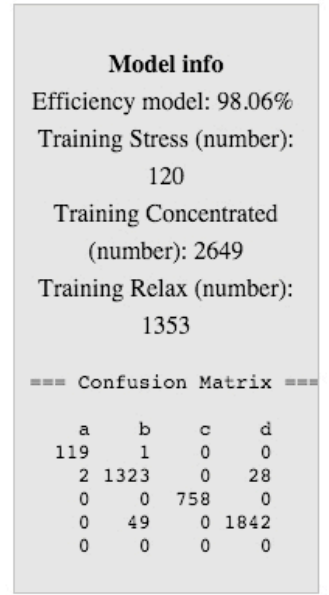

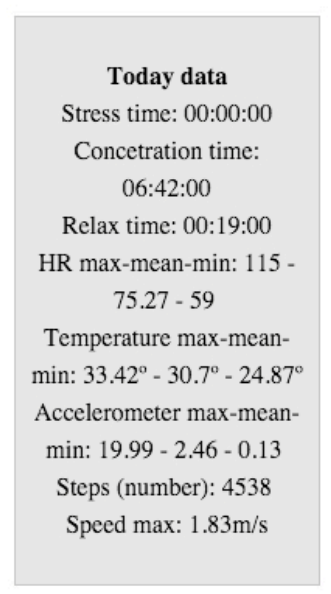

Figure5. Dashboard of stress

\section{Reference architecture}

In addition to the review of wearables currently available on the market and to the identification of indexes from biometric data we initiated the design of a software component architecture to assess the options available to perform data collection from wearables. In this way, the loop needed to develop value added learning analytic solutions based on wearable data is closed. As a starting point architectures already proposed in the learning analytics field were selected: SOLAR (Siemens et al., 2011), DESIRE2LEARN (Essa and Ayad, 2012) and ALAS-KA (Ruipérez-Valiente, Muñoz-Merino and Kloos, 2013). These architectures involve a modular distribution to facilitate the development of analysis tools on learners' data collected from Learning Management Systems (LMS) or Student Information Systems (SIS). The architecture proposed in our piece of research is similar, but focusing on data collected from current mobile devices to expand the potential of the applications and improve the results. In essence, the reference architecture focuses on the achievement of the following operations:

- To request and collect data from mobile and wearable devices.

- To perform data analysis on the data to generate subject behavior patterns and indexes.

- To store the raw data and the results obtained from the analytics and to support data visualization.

The proposed reference architecture is shown in Figure 6. As it can be observed, the upper abstraction level distinguishes three main elements: (i) devices where data is collected; (ii) the analytic engine where the data is processed; and (iii) external agents consuming the analytic results. Next sections describe these elements in more detail. 


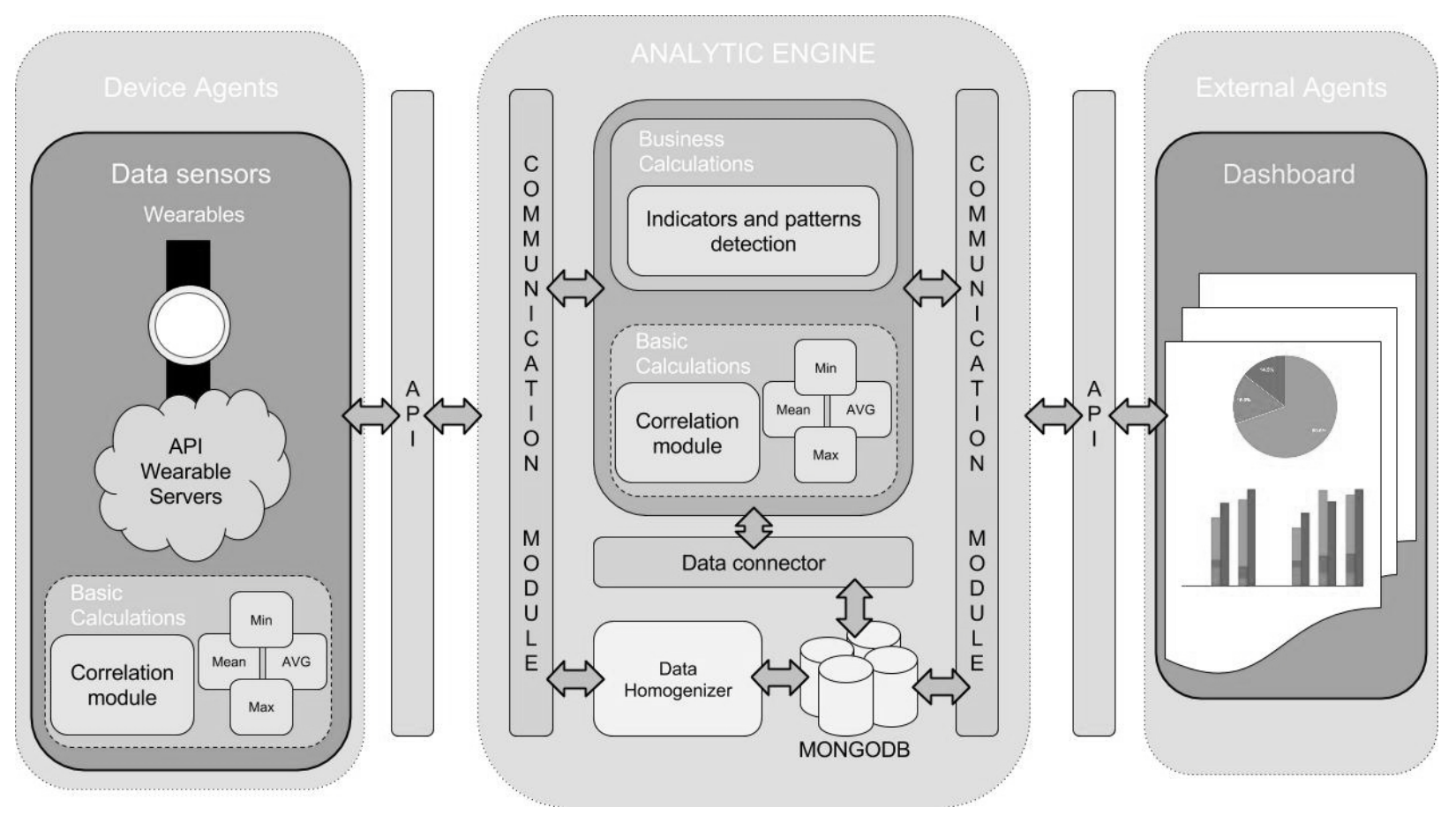

Figure 6. Our architecture

\section{Data collection devices}

The first high level element of the architecture involves the devices collecting data such as the mobile devices, and more specifically the wearables, used by learners. These devices usually include several sensors to capture the data that is stored and eventually sent to an external server owned by the device developer. To obtain the data the analytic engine can get access to the mobile device directly, or more commonly, to contact to the external server. The second option involves several advantages: data is usually already processed by developer algorithms, the access is very simple and independent of the hardware of the device. Nevertheless, it also involves some drawbacks: raw sensors' data cannot be obtained, data is not provided in real time and permission rights needs to be managed (authentication and tokens).

Some of the devices in which data is generated can be programmed. In this way it is possible to develop native apps to perform some kind of processing over the data, even significant parts of an analytic algorithm. As a result, the computational load involved in the analytics processing can be distributed and the load can be balanced to facilitate the system scalability. In the figure a "Basic Calculation" module is included inside the external agent to represent the pieces of software that can be installed in wearables and other mobile devices to perform some calculations. Usually, smartbands cannot support this kind of pre-processing either because they have no option available to install new apps or because the energy demanded is too high.

\section{Analytic engine}

The key element in the reference architecture is the Analytic Engine. This element is devoted to collect and homogenize data from different devices and subjects (students in our case) to perform analytic processes and obtain information from raw data that can be provided to external agents.

Despite it is not the focus of this paper, this element does not just involve the processing of algorithms to obtain the aforementioned indexes, but also the comparative analysis of features and behaviors to get all type of features and behaviors (e.g. social interaction patterns) and to detect outliers.

The Analytic Engine is made up by several modules and submodules for analysis and storage:

\section{Communication module}

This module is responsible for the data communication through external invocations to available APIs. In addition, it is responsible to perform the automatic collection of data. It is made up by three sub-modules: 
API controller, managing the particular logic of each API; Timer Agent, managing the alarms to trigger the requests; and Request Handler, managing the request at high level and intermediating between the former two modules.

\section{Data homogenizer}

This module performs the input data normalization based on some programming rules to generate a uniform and unique data model. In addition, this module relates data collected from several devices referred to the same subject.

\section{MongoDB - warehouse}

It stores raw and processed data. MongoDB was selected as non relational database to store this information because it shows better performance in search time and manages JSON models directly. Particularly:

- It enables searches in a minimum log time and generates fictitious index "search keys" that can be used to collect information of several days or even weeks from a certain user in just some seconds. Its search speed provides results almost in an instantaneous way, improving the analysis response achieving on demand results.

- The JSON structure, as an alternative to the classic XML, allows to arrange the information following a key value model without the need to specify the value type stored. This facilitates the creation of models.

\section{Data connector}

The main task of this module is to connect the stored information in the MongoDB database to the analysis modules. This module includes optimized MongoDB requests to satisfy the needs of the calculation modules.

\section{Basic calculations engine}

This performs the basic and horizontal algebraic and statistical calculations. It is made up by several modules of libraries to perform operations such as minimum value, maximum value, medium value, median value or matrix operations in large data sets efficiently. In some cases, some parts of these calculations can be performed in the mobile devices.

\section{Business calculations engine}

This module performs the analytical calculations to extract patterns and indexes featuring students. They provide not just the indexes described in section 4, but also to perform useful operations for the external agents, such as participant clustering, similarity measures between two learners or, in a more generic way, behavior sequences (in accordance to different factors and criteria) or outlier detection in a group of individuals.

\section{External agents}

The External Agent element represents any system consuming the services provided by the Analytics Engine to offer, in turn, value added services to its own users. Next some examples of external agents are introduced:

- Direct or indirect recommenders. Based on the indexes and patterns that feature learners these systems provide recommendations for educational resources, activities, group pairings, etc. The stress level of a certain learner while performing different activities can be considered to recommend activities.

- Alerting systems that notify to users when some problem or anomaly is detected. For example, when a learner has sleeping difficulties, or when a learner is sleeping in a very different way to the one recognized by his chronotype.

- Measuring systems that assess the quality of educational resources or activities. Learning analytics can process data about results obtained by the learners that have performed a certain activity or used a certain resource to provide information about the quality and other properties of such elements. 
- Dashboards offering learners' data and analytic results for specialized academic roles (e.g. teachers, principals, instructional experts) to provide a clear picture of what is happening during the development of educational activities. They are display tools offering specific tools to support the user making sense of the information. Using this kind of tools, users can get access to key data collected and estimated values in the analytic processing.

\section{CONCLUSIONS}

The domain defined by the application of Information and Communication Technologies (ICT) to education shows a clear interest by the data analytics techniques which goal is to extract information to better understand students and other subjects involved in the educational processes. Learning Analytics is a booming discipline in which many researchers have embarked given its potential to improve the educational processes. As a discipline it is very young and new developments and proposals are wait in the next years. The work included in this paper introduces new opportunities to carry out some developments. More specifically, it contributes its two cents in this field through the definition of a holistic framework that facilitates the exploitation of data collected by wearable devices. The increasing adoption and use of these devices by learners opens the opportunity to further developments in this still little explored field.

The work presented in this paper aims to cover the three basic building blocks needed to develop final systems oriented to support wearable data analysis, namely: features of the wearable devices used to collect data, identification of the useful data elements to be used in the development of valued added services, and definition of a reference architecture to be used as blueprint in the development of eventual systems. These three aspects have been analyzed from a consistent point of view to provide an initial validation of the ideas. At this time, we have already an analytic engine prototype developed in accordance to the reference architecture that is able to collect data from wearables of different developers (managing the issues involved in each case) to provide analytic results and services for third party applications. As a proof of concept of the external agent we also have developed a dashboard showing data collected from students and features related to sleep and stress. Now our research group is working towards the identification of new indexes and also preparing tests in real environments to validate the results obtained so far.

\section{ACKNOWLEDGEMENTS}

Work supported by the European Regional Development Fund (ERDF) and the Galician Regional Government under agreement for funding the Atlantic Research Center for Information and Communication Technologies (AtlantTIC).

\section{REFERENCES}

Ávila, S.M.G., Garzón, L. and Casallas, L.H.C., 2011. Revision de dispositivos electronicos para la determinacion de estres a partir de variables fisiologicas. Visión Electrónica: algo más que un estado sólido, 5(1), pp. 114-122.

Baker, R.S. and Inventado, P.S., 2014. Educational data mining and learning analytics. In Learning Analytics (pp. 61-75). Springer New York.

Bratko, I., Michalski, R.S. and Kubat, M., 1999. Macbine learning and data mining: methods and applications.

Buysse, D.J., Reynolds, C.F., Monk, T.H., Berman, S.R. and Kupfer, D.J., 1989. The Pittsburgh Sleep Quality Index: a new instrument for psychiatric practice and research. Psycbiatry research, 28(2), pp. 193-213.

Cano-Vindel, A. and Miguel-Tobal, J.J., 1999. Evaluación de la ansiedad desde un enfoque interactivo y multidimensional: El inventario de Situaciones y Respuestas de Ansiedad (ISRA). Psicologia Contemporánea,6(1), pp.14-21.

Carskadon, M.A., Dement, W.C., Mitler, M.M., Roth, T., Westbrook, P.R. and Keenan, S., 1986. Guidelines for the multiple sleep latency test (MSLT): A standard measure of sleepiness. Sleep, 9(4), pp. 519-524.

Chen, H., Chiang, R.H. and Storey, V.C., 2012. Business intelligence and analytics: From big data to big impact. MIS quarterly, 36(4), pp. 1165-1188.

Cohen, S., Kamarck, T. and Mermelstein, R., 1983. A global measure of perceived stress. Journal of health and social behavior, pp. 385-396.

Crabtree, I.B. and Rhodes, B., 1998. Wearable computing and the remembrance agent. BT Tecbnology Journal, 16(3), pp. 118-124.

de Arriba Perez, F., Caeiro Rodriguez, M. and Santos Gago, J.M., 2015, June. Knowledge extraction from usage data of mobile devices with educational purposes. In Information Systems and Tecbnologies (CISTI), 2015 10th Iberian Conference on (pp. 1-4). IEEE. 
Essa, A. and Ayad, H., 2012. Improving student success using predictive models and data visualisations. Research in Learning Technology, 20.

Fayyad, U.M., Piatetsky-Shapiro, G., Smyth, P. and Uthurusamy, R., 1996. Advances in knowledge discovery and data mining.

Horne, J.A. and Ostberg, O., 1975. A self-assessment questionnaire to determine morningness-eveningness in human circadian rhythms. International journal of chronobiology, 4(2), pp. 97-110.

ITU Telecom World 2015, 2015. Dirigentes de las TIC mundiales compartirán ideas en ITU Telecom World 2015. Available at: http://www.itu.int/net/pressoffice/press_releases/2015/40-es.aspx (Accessed: February 20, 2016).

Johns, M.W. and others, 1991.A new method for measuring daytime sleepiness: The Epworth sleepiness scale. Sleep, 14(6), pp. 540-545.

LAK2011, 2010. Call for Papers | 1st International Conference on Learning Analytics and Knowledge 2011. Available at: (Accessed: February 17, 2016).

Manyika, J., Chui, M., Brown, B., Bughin, J., Dobbs, R., Roxburgh, C. and Byers, A.H., 2011. Big data: The next frontier for innovation, competition, and productivity.

Maslach, C. and Jackson, S.E., 1981. The measurement of experienced burnout. Journal of organizational behavior, 2(2), pp. 99-113.

Maslach, C., Jackson, S.E. and Leiter, M.P., 1997. Maslach burnout inventory. Evaluating stress: A book of resources, 3 , pp.191-218.

Mayer-Schönberger, V. and Cukier, K., 2013. Big data: A revolution that will transform how we live, work, and think. Houghton Mifflin Harcourt.

Pacheco, N.E., Durán, M.A.D. and Rey, L., 2007. Inteligencia emocional y su relación con los niveles de burnout, engagement y estrés en estudiantes universitarios. Revista de educación, (342), pp.239-256.

Picard, W. and Healey, J.A., 2000. Wearable and automotive systems for affect recognition from physiology.

Rehman, M.H.u., Liew, C.S., Wah, T.Y., Shuja, J. and Daghighi, B., 2015. Mining personal data using smartphones and wearable devices: A survey. Sensors, 15(2), pp. 4430-4469. doi: 10.3390/s150204430.

Richter, F., 2015. The Predicted Wearables Boom Is All About The Wrist | Statista. Available at: http://www.statista.com/chart/3370/wearable-device-forecast/ (Accessed: February 16, 2016).

Romero, C. and Ventura, S., 2013. Data mining in education. Wiley Interdisciplinary Reviews: Data Mining and Knowledge Discovery, 3(1), pp. 12-27.

Romero, C. and Ventura, S., 2010. Educational data mining: a review of the state of the art. Systems, Man, and Cybernetics, Part C: Applications and Reviews, IEEE Transactions on, 40(6), pp.601-618.

Ruipérez-Valiente, J.A., Muñoz-Merino, P.J. and Kloos, C.D., 2013, November. An architecture for extending the learning analytics support in the Khan Academy framework. In Proceedings of the First International Conference on Technological Ecosystem for Enhancing Multiculturality (pp. 277-284). ACM.

Russom, P., 2011. Big data analytics. TDWI Best Practices Report, Fourth Quarter, pp.1-35.

Santos Sierra, A.D., 2012. Design, implementation and evaluation of an unconstrained and contactless biometric system based on band geometry and stress detection (Doctoral dissertation, Telecomunicacion).

Shum, S.B. and Ferguson, R., 2012. Social learning analytics. Educational technology \& society, 15(3), pp. 3-26.

Siemens, G., Gasevic, D., Haythornthwaite, C., Dawson, S., Shum, S.B., Ferguson, R., Duval, E., Verbert, K. and Baker, R., 2011. Open Learning Analytics: an integrated \& modularized platform.

Swan, M., 2012. Sensor Mania! The Internet of Things, Wearable Computing, Objective Metrics, and the Quantified Self 2.0. Journal of Sensor and Actuator Networks, 1(3), pp. 217-253. doi: 10.3390/jsan1030217.

Tehrani, K. and Michael, A., 2014. Wearable technology and wearable devices: Everything you need to know. Wearable Devices Magazine.

UNESCO, 2015. ICT in Education | United Nations Educational, Scientific and Cultural Organization. Available at: http://www.unesco.org/new/en/unesco/themes/icts/ (Accessed: February 16, 2016).

Zhai, J. and Barreto, A., 2006. Stress detection in computer users based on digital signal processing of noninvasive physiological variables. Engineering in Medicine and Biology Society, 2006. EMBS'06. 28th Annual International Conference of the IEEE, pp. 1355-1358. 